\title{
On Fuzz of Spun Yarns
}

\section{Part 1: Effects of Structure of Spun Yarns on Their Fuzz}

\author{
By Kenji Tanaka, Member, TMSJ \\ Nagoya Institute of Technology, Nagoya
}

\begin{abstract}
The resistance of fibres to slippage out of spun yarns has an important bearing on the weavability of yarns, fuzzing and pilling.

In this study, the theoretical relation between this force and the geometrical build of yarns has been obtained and it has been ascertained that it has a high correlation with the experimental results on fuzz generation.
\end{abstract}

\section{Introduction}

Since 1950, investigations into the fuzz of spun yarns have been made by Onions $[1,2,3]$, Pickering [1], Stables[1], Yate[2], Lappage[3'], Boswell[7], Townend[7], Barella $[4,5]$, Centre de Recherches des Industries Textile de Rouen[6], Mihira[8], Sugimoto[8], the Japan Cotton Technical Institute et al. They are mostly related to measuring and evaluating the fuzz of yarns, and inquiry into factors generating fuzz during spinning.

A spun yarn which develops much fuzz during spinning is believed to develop fuzz easily by surface rubbing during use. Fuzz generated by surface rubbing is a major problem in subsequent processing and has an important bearing upon the appearance of cloth. Fuzz depends not only on the physical properties of fibres but on the geometrical structure of yarns. Most of the published researches evaluates the relation between the physical properties of fibers and the geometrical build of yarns by experimental estimation.

There are few, if any, published works which evaluate this relation theoretically.

This article deduces, from the geometrical build of yarns, the power of resistance of fibers to slippage out of yarns - this slippage is a major contributing factor to the fuzz of yarns - and presents the results of experiments which have shown that theoretical resistance power is useful for evaluating fuzz.

\section{Resistance Power of Fibers against Slip- page out of Spun Yarns}

The degree of the tendency of fuzz to develop in spun yarns is governed by the resistance power of fibers against slippage out of yarns. To figure out this resistance power, we must figure out the pressure generated in all parts of a yarn while an amount of tension acts on the yarn. We can then deduce the resistance power of fibers against slippage out of the yarn if we integrate the resistance power of all the fine parts of fibers migrating in a spun yarn.

(1) Pressure inside spun yarns

It would be extremely difficult to measure pressure in a spun yarn from its structure if we paid attention to the migration of fibres in the yarn, but the migration of fibers is so limited that its effects may be ignored. The author has measured internal pressure on the assumption that a yarn consists of fibres wound spirally on concentric cylinder surfaces.
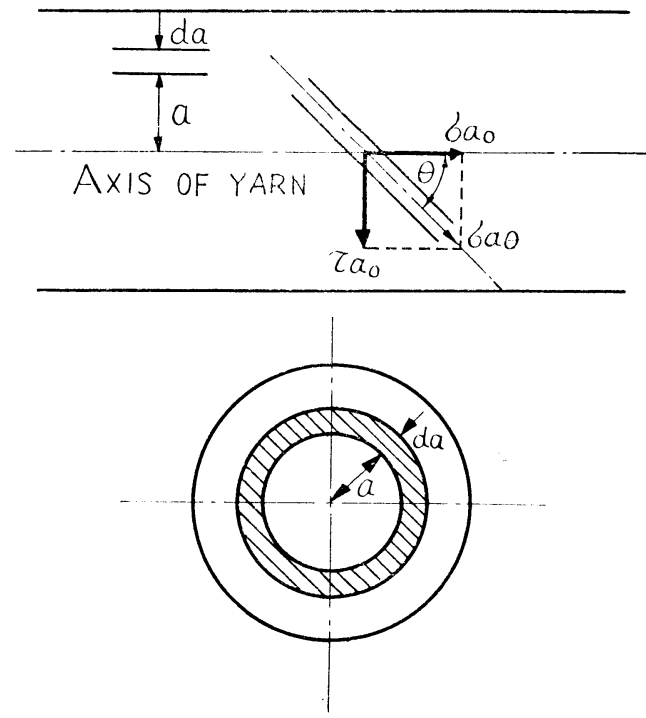

Fig. 1 Stress on twisted yarn and its constituent fibres 
Tensile stress $\sigma_{a}$ in the direction of the fibre axis in Fig. 1 having spiral angle $\theta$ can be divided into tensile stress $\sigma_{a 0}$ in the direction of yarn axis and shearing stress $\tau_{a 0}$ in a perpendicular plane to the yarn axis. Therefore, the following equation forms :

$$
\left.\begin{array}{l}
\sigma_{a 0}=\sigma_{a g} \cdot \cos ^{2} \theta \\
\tau_{a 0}=12 \sigma_{a} \cdot \sin 2 \theta
\end{array}\right\}
$$

Tensile force $d F_{0}$ parallel to the yarn axis in the shaded annular ring at distance $a$ from the yarn axis is expressible thus :

$$
\left.d F_{0}=2 \pi a \cdot \varphi \cdot \cos ^{2} \theta \cdot \sigma_{a}\right) \cdot d a
$$

where $\varphi$ is the cover factor of the yarn (fullness of fibres in the yarn).

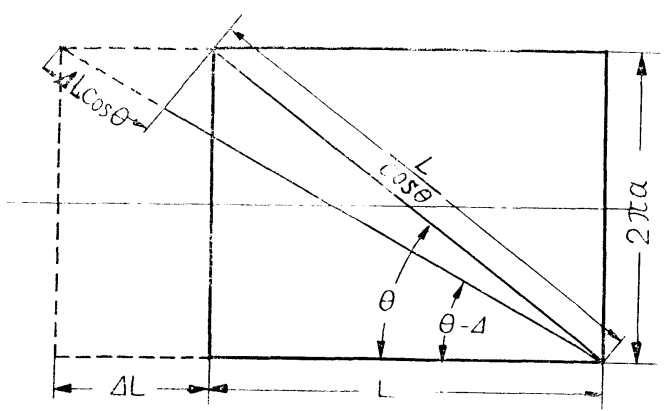

Fig. 2 Deformation of twisted yarn

Elongation $\varepsilon_{f}$ of fibres in Fig. 2 having spiral angle $\theta$ is aproximately expressible as follows :

$$
\varepsilon_{f} \doteqdot \frac{\Delta L \cdot \cos \theta}{L / \cos \theta}=\frac{\Delta L}{L} \cdot \cos ^{2} \theta
$$

The strain being small, approximately $\sigma_{a^{\prime}}=\kappa \cdot \varepsilon_{f}$ ( $\kappa$ is the elastic constant of fibers).

The relation between spiral angle $\theta$ of fibres at distance $a$ from the yarn axis and the number $N$ of twists per unit length of yarn is expressible thus :

$$
\cos ^{2} \theta=\frac{1}{4 \pi^{2} N^{2} a^{2}+1}
$$

Therefore, the following equations emerge, assuming coverfactor $\varphi=1$,

$$
\begin{aligned}
& \varepsilon_{f}=\frac{\varepsilon_{y}}{4 \pi^{2} N^{2} a^{2}+1} \\
& F_{u}=\int d F_{0}=\frac{\pi \kappa a_{0}{ }^{2} \cdot \varepsilon_{y}}{4 \pi^{2} N^{2} a_{0}{ }^{2}+1} \\
& \therefore \sigma_{a}=\kappa \cdot \varepsilon_{f}=\frac{\kappa \cdot \varepsilon_{y}}{4 \pi^{2} N^{2} a^{2}+1}=\frac{F_{0}}{\pi a_{0}{ }^{2}} \cdot \frac{4 \pi^{2} N^{2} a_{0}{ }^{2}+1}{4 \pi^{2} N^{2} a^{2}+1} \cdots(5)
\end{aligned}
$$

where $\varepsilon_{y}(=\Delta L / L)$ is the tensile strain of yarn; $a_{0}$ is the outside radius of yarn and $F_{0}$ is the yarn tension.

The radius of curvature $\rho_{a}$ of fibres wound spirally on the surface of a cylinder which has radius $a$ is :

$$
\rho_{a}=\frac{a}{\sin ^{2} \theta}-\frac{4 \pi^{2} N^{2} a^{2}+1}{4 \pi^{2} N^{2} a}
$$

Since inside pressure $d P$ produced by the annular ring ( $d a$ in thickness) in radius $a$ of a yarn is $\frac{\sigma_{a} \theta}{\rho_{a}} \cdot d a$, internal pressure $P_{a}$ at distance $a$ from the yarn axis is expressible thus:

$$
\begin{aligned}
& P_{a}=\int d P=\int_{a}^{a_{0}} \frac{\sigma_{a^{\prime}}}{\rho_{a}} d a \\
& =\frac{F_{0}\left(4 \pi^{2} N^{2} a_{0}{ }^{2}+1\right)}{2 \pi a_{0}{ }^{2}} \cdot\left(\frac{1}{4 \pi^{2} N^{2} a^{2}+1}-\frac{1}{4 \pi^{2} N^{2} a_{0}{ }^{2}+1}\right)
\end{aligned}
$$

Therefore, internal pressure $P_{0}$ in the center of the yarn is:

$$
P_{0}=\lim _{a \rightarrow 0} \int_{a}^{a_{0}} \frac{\left.\sigma_{a}\right)}{\rho_{a}} \cdot d a=2 \pi N^{2} F_{0}
$$

(2) Resistance of Fibres to Slippage out of Spun Yarns

Now to deduce the power of resistance of fibre to slippage out of fibers migrating radial displacement at the rate of $r_{1}$ per pitch of spiral. First, find out the elementary length of fiber $d l$ wound spirally on a cylin. der of variable radius $a$ with rotating angle $t$ along the yarn axis. In figure 3 , the coordinates of any point on the spiral are as follows,

$$
\left.\begin{array}{l}
x=a(t) \cdot \cos \mathrm{t} \\
y=a(t) \cdot \sin \mathrm{t} \\
z=t / 2 \pi N
\end{array}\right\}
$$

where

$t=$ rotating angle of radius $a$ of the spiral cylinder with a fiber wound on its surface

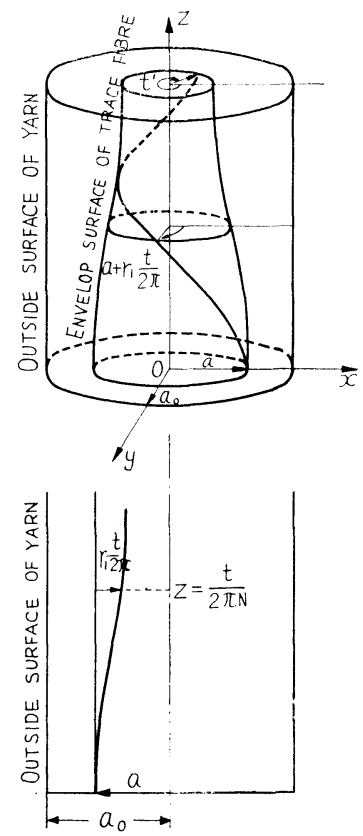

Fig. 3 Spacial spiral curve and its enveloped surface drawn by the fiber on the surface of twisted yarn 
$a(t)=$ radius of the spiral cylinder

Assume :

$$
a(t)=a_{1}+r_{1} \frac{t}{2 \pi}
$$

Then :

$$
\left.\begin{array}{l}
x=\left(a_{1}+r_{1} \frac{t}{2 \pi}\right) \cdot \cos \mathrm{t} \\
\left.y=a_{1}+r_{1} \frac{t}{2 \pi}\right) \cdot \sin \mathrm{t} \\
z=t / 2 \pi N
\end{array}\right\}
$$

The relation between rotating angle $d t$ of radius $a$ of the spiral cylinder and length $d l$ of the fiber wound on its surface is expressible thus :

$$
\begin{aligned}
d l & =\sqrt{\dot{x}^{2}+\dot{y}^{2}+\dot{z}^{2}} \cdot d t \\
& =\frac{1}{2 \pi N} \sqrt{N^{2}\left(2 \pi a_{1}+r_{1} t\right)^{2}+N^{2} r_{1}{ }^{2}+1} \cdot d t
\end{aligned}
$$

Assuming :

$\lambda=$ length of perimeter of fibre's section $\mu=$ frictional coefficient of fibres

then the frictional resistance received by fine part $d l$ of the fibre, i.e., the power of resistance of the fibre to slippage is :

$$
d Q=\mu \cdot \lambda \cdot P_{a} \cdot d l
$$

The resistance power of fibres migrating in a yarn to slippage per mean reversing period (rotating angle $\Theta$ of radius $a$ of the spiral cylinder) is expressible as follows :

$$
\begin{aligned}
Q= & \int_{0}^{\ominus} \frac{\mu \lambda F_{0} \sqrt{ } 4 \pi^{2} N^{2} a^{2}+N^{2} r_{1}{ }^{2}+1}{4 \pi^{2} N a_{0}{ }^{2}} \\
& \cdot\left(\frac{4 \pi^{2} N^{2} a_{0}{ }^{2}+1}{4 \pi^{2} N^{2} a^{2}+1}-1\right) d t
\end{aligned}
$$

From $a=a_{0}-r_{1} \frac{t}{2 \pi}$ it is apparent that :

$$
d t=-\frac{2 \pi}{r_{1}} \cdot d a
$$

Therefore :

$$
\begin{aligned}
& Q=-\frac{\mu \lambda F_{0}}{2 \pi N a_{0}^{2} r_{1}} \int_{a_{0}}^{a_{0}-r_{1-\frac{\Theta}{2 c}}} \sqrt{4 \pi^{2} N^{2} a^{2}+N^{2} \overline{r_{1}{ }^{2}+1}} \\
& \cdot\left(\frac{4 \pi^{2} N^{2} a_{0}{ }^{2}+1}{4 \pi^{2} N^{2} a^{2}+1}-1\right) \cdot d a \\
& =\frac{\mu \lambda F_{0}}{4 \pi^{2} N a_{0}^{2}}\left(4 \pi^{2} N^{2} a_{0}{ }^{2}+1\right) \\
& \left\{\tan ^{-1} \frac{2 \pi N^{2} r_{1} a_{0}}{\sqrt{4 \pi^{2} N^{2} a_{0}{ }^{2}+N^{2} r_{1}{ }^{2}+1}}\right. \\
& -\tan \frac{2 \pi \cdot N^{2} r_{1}\left(a_{0}-r_{1} \frac{\Theta}{2 \pi}\right)}{\sqrt{4 \pi^{2} N^{2}\left(a_{0}-r_{1} \frac{\Theta}{2 \pi}\right)^{2}+N^{2} r_{1}{ }^{2}+1}} \\
& +\frac{1}{N r_{1}} \cdot \ln \frac{2 \pi N a_{0}}{2 \pi N\left(a_{0}-r_{1} \frac{\Theta}{2 \pi}\right)}
\end{aligned}
$$

$$
\begin{gathered}
\left.\frac{+\sqrt{4 \pi^{2} N^{2} a_{0}^{2}+N^{2}{\overline{r_{1}}}^{2}+1}}{+\sqrt{4 \pi^{2} N^{2}\left(a_{0}-r_{1} \frac{\Theta}{2 \pi}\right)^{2}+N^{2} r_{1}{ }^{2}+1}}\right\} \\
-\frac{\mu \lambda F_{0}}{4 \pi^{2} N a_{0}^{2} r_{1}}\left\{\pi a_{0} \sqrt{4 \pi^{2} N^{2} a_{0}{ }^{2}+N^{2} r_{1}{ }^{2}+1}\right. \\
-\pi\left(a_{0}-r_{1} \frac{\Theta}{2 \pi}\right) \sqrt{4 \pi^{2} N^{2}\left(a_{0}-r_{1} \frac{\Theta}{2 \pi}\right)^{2}+N^{2} r_{1}{ }^{2}+1} \\
+\frac{N^{2} r_{1}{ }^{2}+1}{2 N} \cdot \ln \frac{2 \pi N a_{0}}{2 \pi N\left(a_{0}-r_{1} \frac{\Theta}{2 \pi}\right)} \\
\left.\frac{+\sqrt{4 \pi^{2} N^{2} a_{0}{ }^{2}+N^{2} r_{1}{ }^{2}+1}}{\left.+\sqrt{4 \pi^{2} N^{2}\left(a_{0}-r_{1} \frac{\Theta}{2 \pi}\right.}\right)^{2}+N^{2} r_{1}{ }^{2}+1}\right\}
\end{gathered}
$$

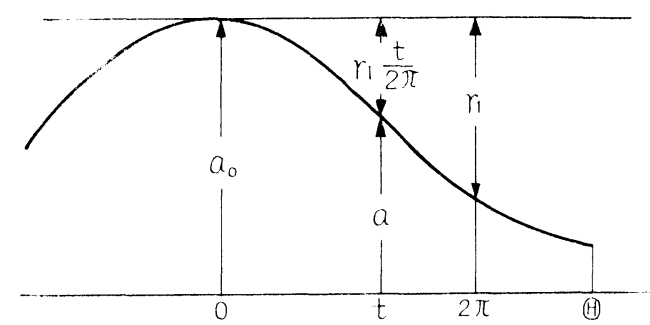

Fig. 4 Developed curve of spiral drawn by the fiber

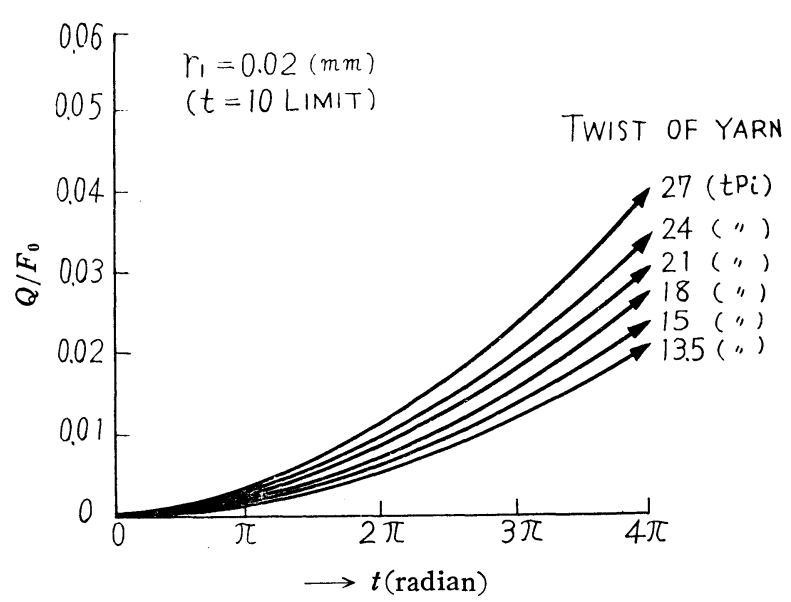

Fig. 5 Relation between rotating angle $t$ of spiral radius $a$ along the fiber length and resistance power of fibres against slippage out of yarns which have different twist $N$, when the migration of fibres is constant

Assuming $r_{1}=0.02 \mathrm{~mm}, a_{0}=0.1 \mathrm{~mm}, \lambda=0.15 \pi \mathrm{mm}$ and $\mu=0.2$, then the relation between $Q / F_{0}$ and $t$ for $N=$ 13.5, 15, 18, 21, 24, 27 tpi is as shown in Fig. 5.

Assuming $N=18 \mathrm{tpi}, a_{0}=0.1 \mathrm{~mm}, \lambda=0.015 \pi \mathrm{mm}$, and $\mu=0.2$, the relation between $Q / F_{0}$ and $t$ for $r_{1}=0.01$, $0.02,0.03,0.04,0.05$ is as shown in Fig. 6. 


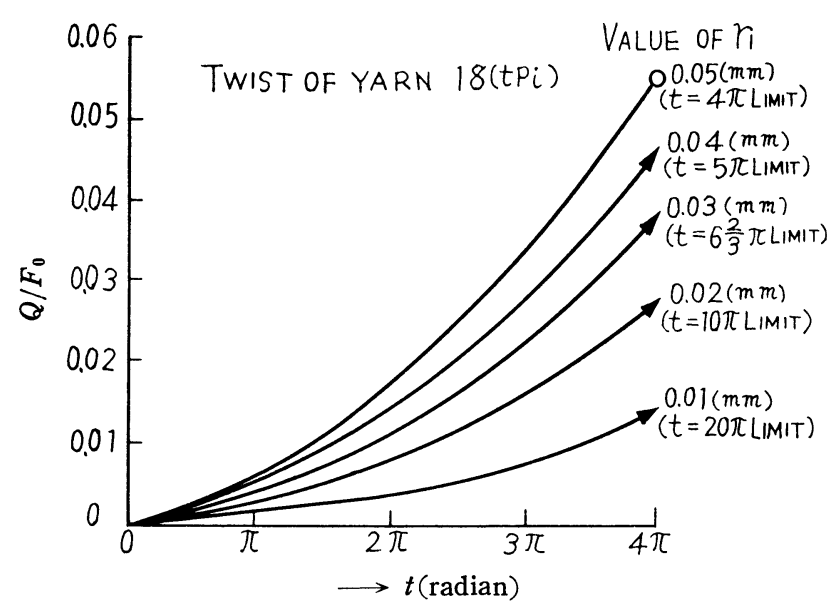

Fig. 6 Relation between $t$ and $Q / F_{0}$ of various yarns which have different migration of fibres respectively under same number of twists of yarns

As is obvious from the diagrams, the resistance power of fibres against slippage per unit rotation angle of radius $a$ of the spiral cylinder increases with an increase in the number of twists of the yarn and in the migration of fibers. To measure the anti-slippage resistance of fibers $l$ in length and migrating at the rate of $r_{1}$ in one reversing period, we must figure out the relation between $l$ and rotating angle $t$ of radius $a$ of the spiral cylinder, or radius $a$ which is the function of $r$. and $t$.

$$
a=a_{0}-r_{1} \frac{t}{2 \pi} \quad \text { Therefore, } d t=-\frac{2 \pi}{r_{1}} \cdot d a
$$

Substituting this equation into eq. (11) gives:

$$
\begin{aligned}
l= & -\frac{1}{N r_{1}} \int_{a_{0}}^{a} \sqrt{4 \pi^{2} N^{2} a^{2}+N^{2} r_{1}{ }^{2}+1} \cdot d a \\
= & \frac{1}{2 N r_{1}}\left(a_{0} \sqrt{4 \pi^{2} N^{2} a_{0}{ }^{2}+N^{2} r_{1}{ }^{2}+1}\right. \\
& -a \sqrt{4 \pi^{2} N^{2} a^{2}+N^{2} r_{1}^{2}+1}+\frac{N^{2} r_{1}{ }^{2}+1}{4 \pi N^{2} r_{1}} \\
& \cdot \ln \frac{2 \pi N a_{0}+\sqrt{4 \pi^{2} N^{2} a_{1}{ }^{2}+N^{2}{r_{1}}^{2}+1}}{2 \pi N a+\sqrt{4 \pi^{2} N^{2} a^{2}+N^{2} r_{1}{ }^{2}+1}} \cdots \cdots \cdots(13)
\end{aligned}
$$

A curve of the relation between $a$ and $l$ can be drawn in the light of eq. (13). $t$ being proportional to $a$ they can be put in the same abscissa, as in Fig. 7.

The length $l$ of fibers for the various values of $N$ when the above-mentioned yarns migrate at the rate of $r_{1}=0.02 \mathrm{~mm}$ is shown in Fig. $8 . \quad l$ for the various values of $r_{1}$ when the number of twists is 18 tpi is shown in Fig. 9.
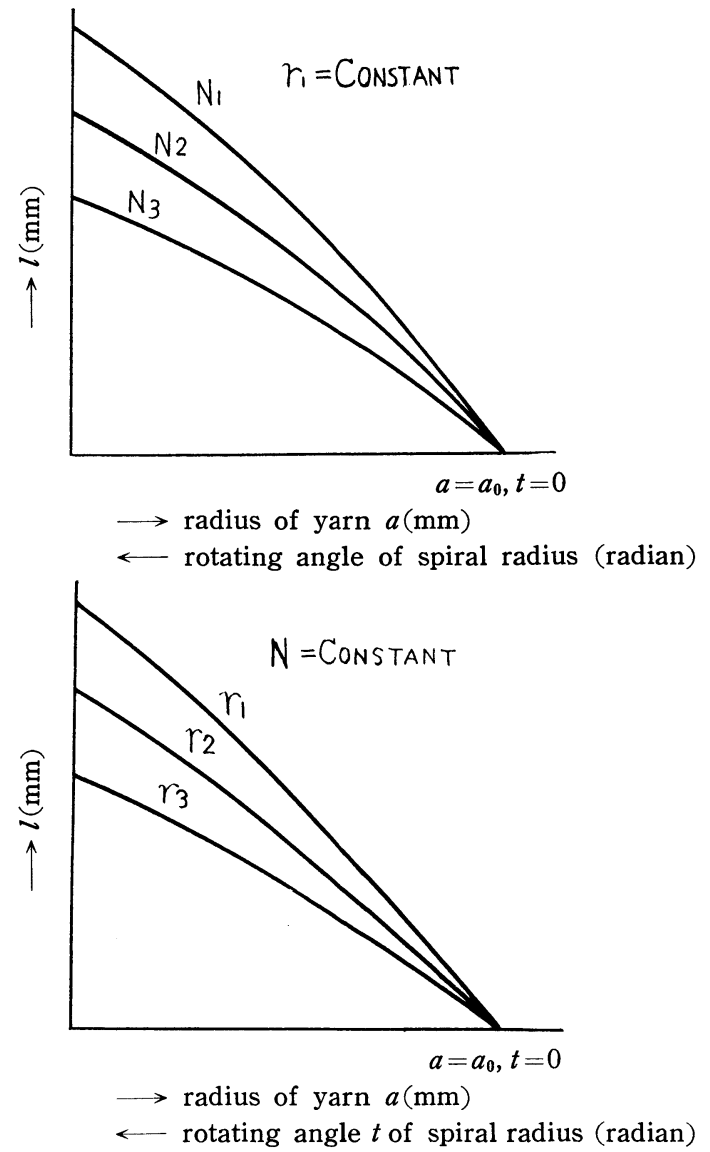

Fig. 7 Relation between length $l$ of fibres, $t$ and radius $a$ in yarns.

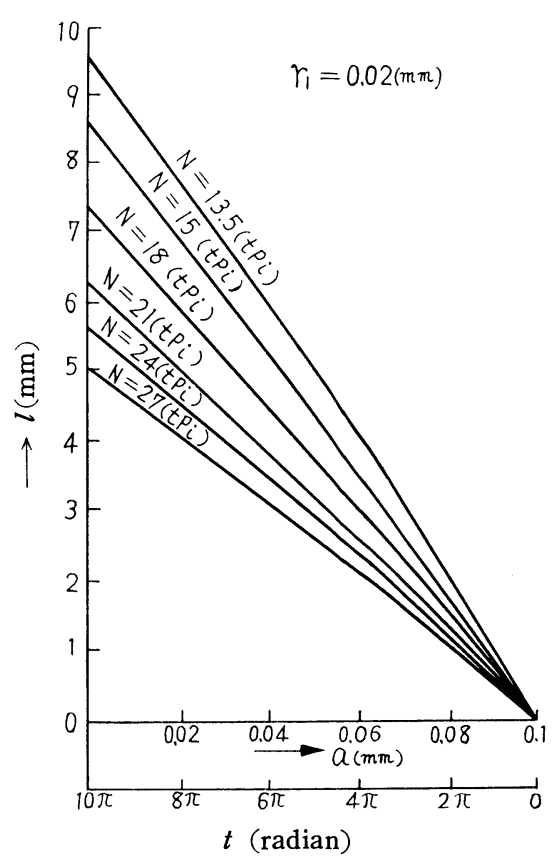

Fig. 8 Concrete example of Fig. 7 


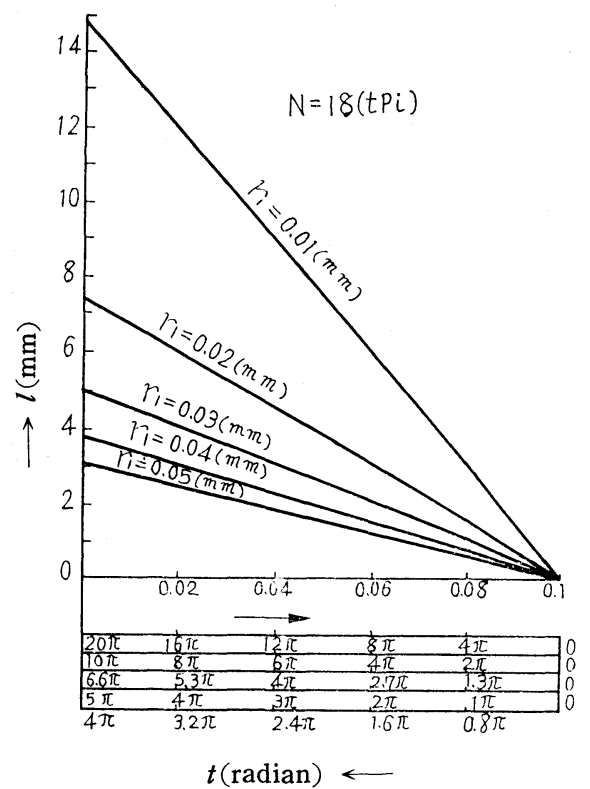

Fig. 9 Concrete example of Fig. 7
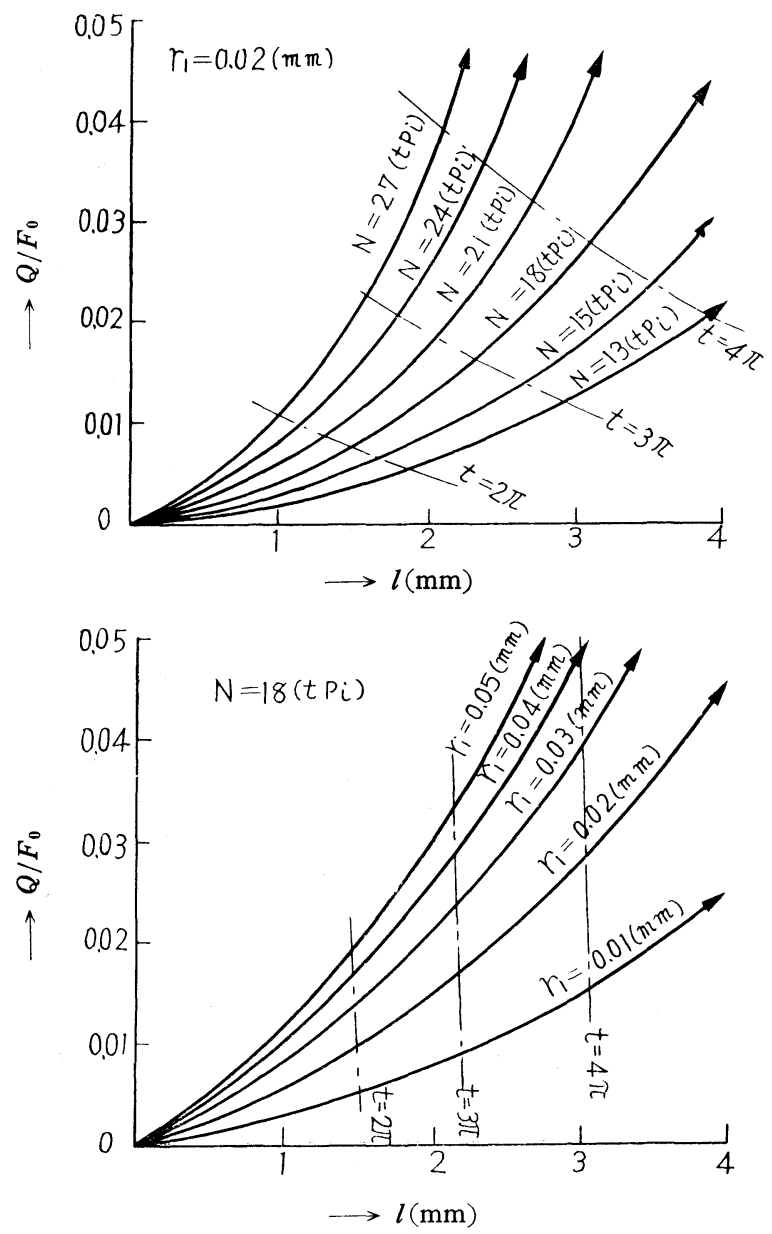

Fig. 10 (A), (B) Relation between length $l$ of fibres pulled out of yarns and $Q / F_{0}$
The relation between $l$ and $Q / F_{0}$ in the abovementioned yarns is shown in Fig. $10(\mathrm{~A})$ and $(\mathrm{B})$. The tendency to generate fuzz out of spun yarn must be estimated from the mean resistence of fiber to slippage out of its unit length. It may be found by dividing the slippage resistance of fibre with length $l$ in reversing period $t$ by fiber length $l$.

The anti-slippage resistance power of fibres for $a_{0}=0.06,008,0.10,0.12$ and $0.14 \mathrm{~mm}$ when $\lambda=0.015 \pi \mathrm{mm}$, $r_{1}=0.02 \mathrm{~mm}, \mu=0.2, N=18 \mathrm{tpi}$, is shown in Figs. 11 (A) and (B).

It is obvious from the diagrams that the antislippage resistance power of fibers decreases as the yarn diameter increases, provided the other factors remain the same. If the fiber length changes, then fiber migration and its reversing period should change as a matter of course.

Assuming the migration of fibers to be constant, the number of fiber ends in a yarn is in inverse ratio to the fiber length and, therefore, the number of hairs ends slipping out — one end of them held in the yarn
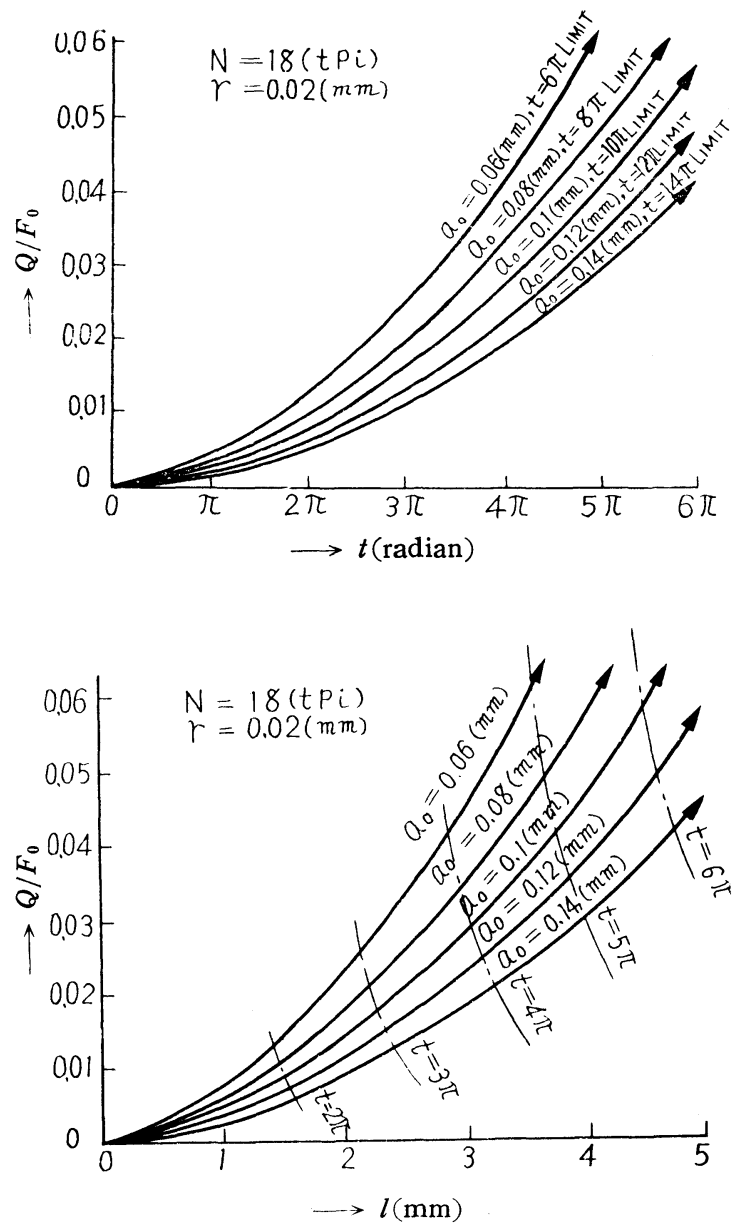

Fig. 11 (A), (B) Relation between $t$ and $Q / F_{0}$ 
- should be in inverse ratio to the fiber length. An increase in the fiber diameter should increase the length of the parameter of the fiber and enhance the anti-slippage re sistance power of fibers.

In practice, however, inter-fiber space increases the fullness of fibers decreases, the fibers become less inter-wound and their anti-slippage resistance power declines.

\section{Procedure of Experiment and Discussion}

(1) The test yarns used in our experiments were 30 s yarns with 13.5, 15, 18, 21, 24 and $27 \mathrm{tpi}$, spun on a Howa Spinlector (spindle revolutions 10,000 rpm), 160 grain/30yd roving, composed of $2 \mathrm{~d} 60-\mathrm{mm}$ rayon staple dull fibers $6 \mathrm{~g}$ in mean strength and $14 \%$ in elongation. (See Fig. 12 for relation between twists, strength and elongation.)
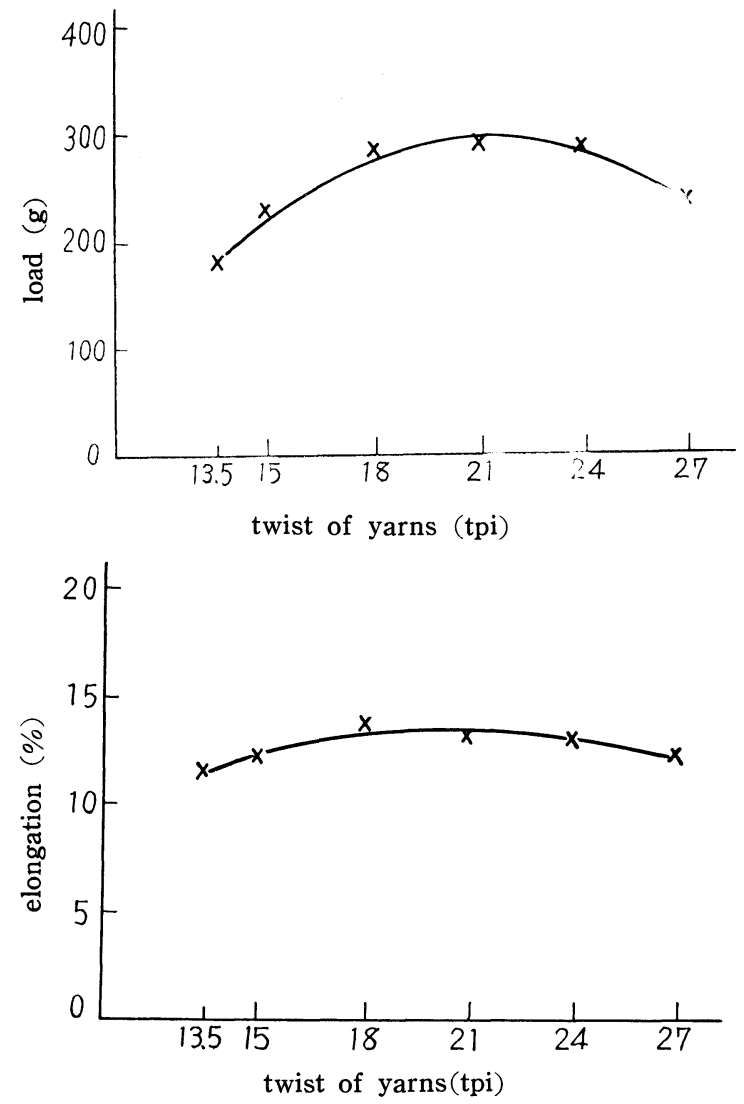

Fig. 12 Load-elongation diagram of $30^{\prime}$ s rayon staple fiber yarns (test yarns)

The test yarns were rubbed on an abrasion tester and their fuzz was measured by photo-microprojector and photoelectric methods. The fuzz was magnified 50 times by the micro-projector method and the shape, length, number, etc. of the hairs observed. The hairs were classified into 2 types for observation. Hairs of type 1 had one end held in the yarns, the other end being left free. Type 2 of hairs had both ends held in the yarns.

During the experiment by the photoelectric method, the test yarns were placed between electrified plates, $1,000-6,000 \mathrm{v}$, and the amount of fuzz was measured by letting the fuzz bristle and absorb pencil light thrown from the side.

The fuzz which generated in a 30 s rayon staple yarn with 18 tpi is shown in Figs. 13 (A) and (B).

Fig. 13 (A) shows the frequency of rubbing and the number of hairs. It shows that hairs increased in number until rubbing was repeated 30 times - and decreased when rubbing was repeated 40 times. This means that excessive rubbing causes the slipping out of fibers, which, in turns, causes hairs to fall out.
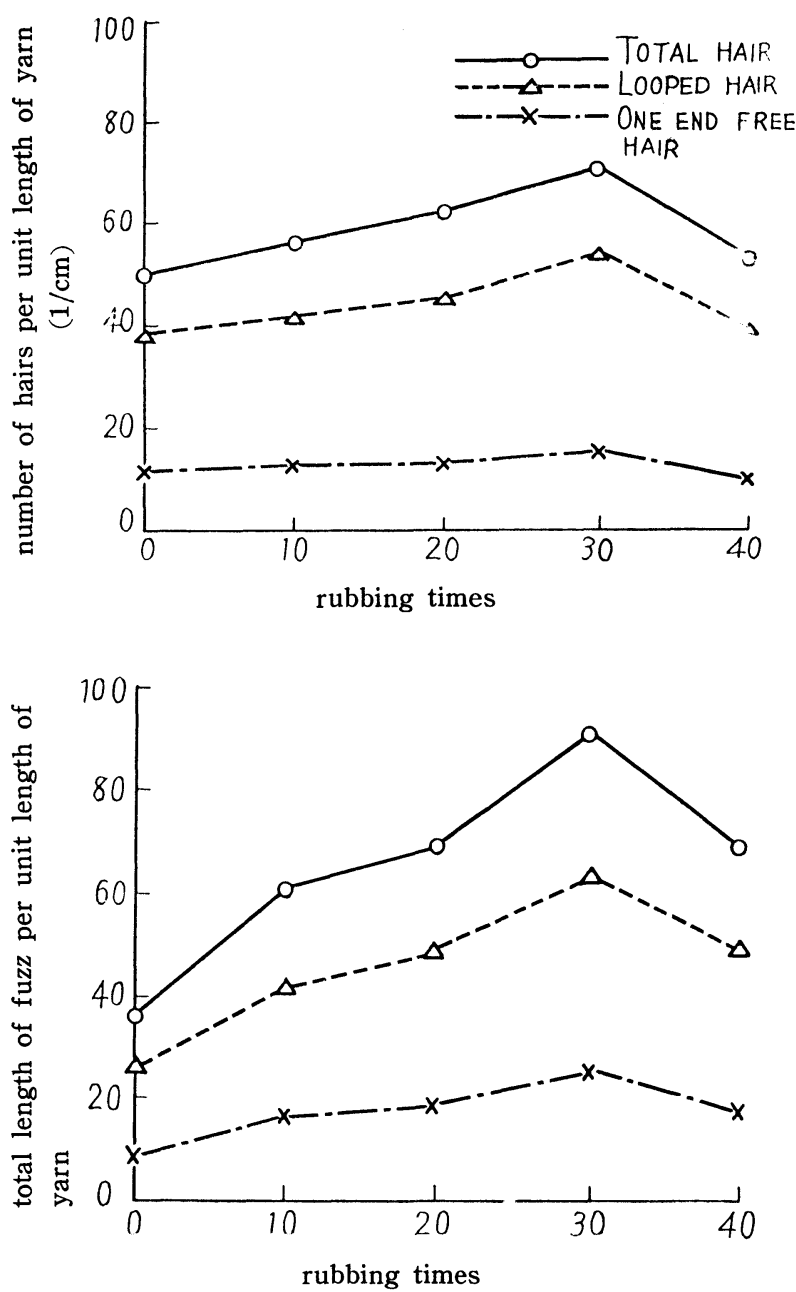

Fig. 13 (A), (B) Results of measurement of fuzz by microscopic method 
Fig. 13 (B) shows the relation between the frequency of rubbing and the total length of fuzz - the overall length of hairs joined end to end. Hairs increased rapidly in number until rubbing was repeated 30 times - and decreased when rubbing was repeated 40 times. This shows that excessive rubbing causes fuzz to fall out.

The generated fuzz measured by the photoelectric method is shown in Figs. 14 (A) and (B). Fig. 14 (A) shows the relation between the frequency of rubbing and the total length of fuzz of test yarns with twists in varying numbers. Fig. 14 (B) shows the relation between the frequency of rubbing and the mean length of fuzz of all the test yarns.

The fuzz of a yarn with 15 tpi increased rapidly
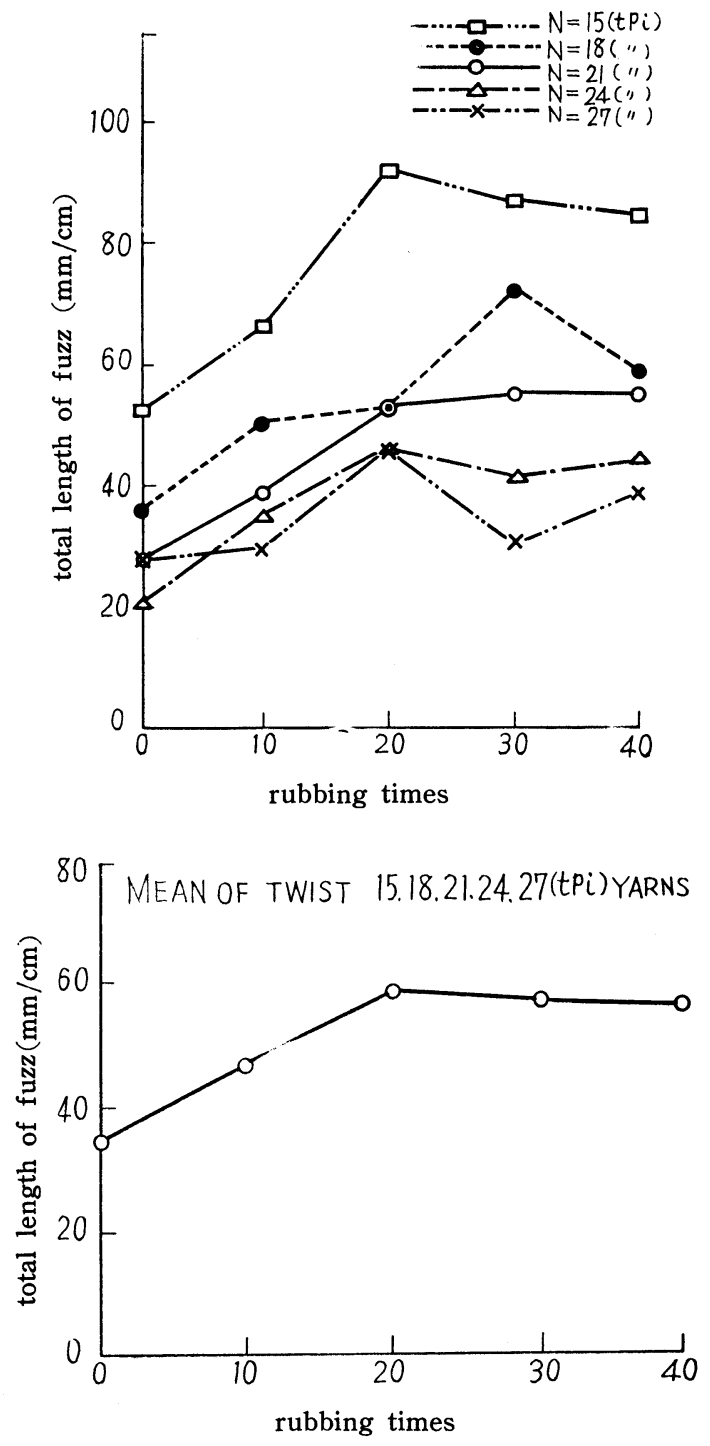

Fig. 14 (A), (B) Results of measurement of fuzz by photoelectric method in amount until rubbing was repeated 20 times. The fuzz of a yarn with 18 tpi increased rapidly in amount until rubbing was repeated 30 times. The fuzz of both yarns decreased in amount when subjected to more rubbing.

The fuzz of test yarns with 21,24 and 27 tpi increased slowly in total length until rubbing was repeated 20 times - and remained practically unchanged in total length when subjected to more rubbing. This means an equilibrium between the generation and falling out of fuzz.

Fig. 15 (A) shows the relation between the number of twists of a yarn and the total length of fuzz rubbed 20, 30 and 40 times. Fig. 15 (B) shows the relation between the number of twists of a yarn and the mean
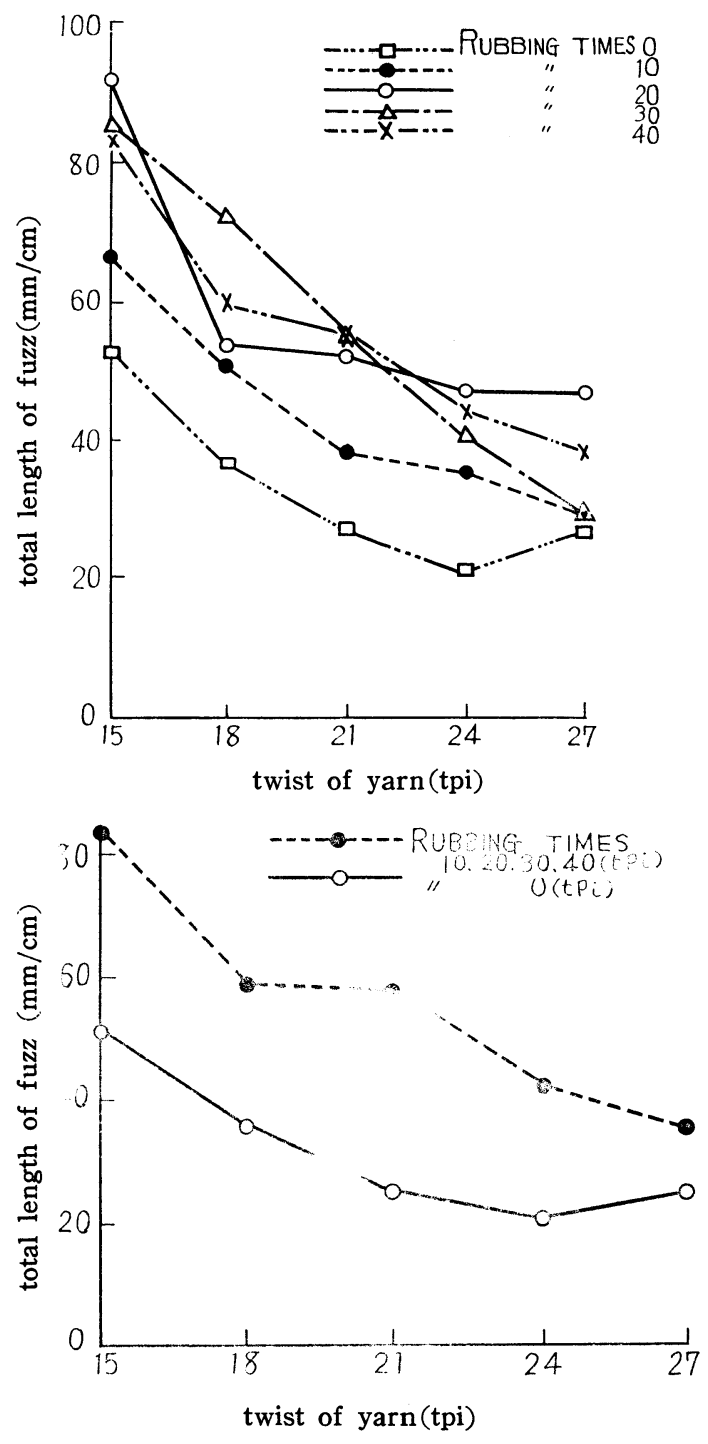

Fig. 15 (A), (B) Results of measurement of fuzz by photoelectric method 
length of fuzz rubbed $10,20,30$ and 40 times.

The larger the number of twists, the more difficult it is for fuzz to generate.

(2) Discussion based on relation between experimental results and theoretical anti-slippage resistance of fibers.

The degree of the tendency of fuzz to generate in yarns can be estimated quantitatively from the power of fibers of an equal length to resist slippage out of the yarns which they constitute. Take, for example, Figs. 10 (A) and (B). It is possible to measure from them the resistance power of fibers of a certain length against slippage out of 30 's yarns.

Assume that the resistance power of fibers $3 \mathrm{~mm}$ long against slippage out of 30 's rayon staple fibers

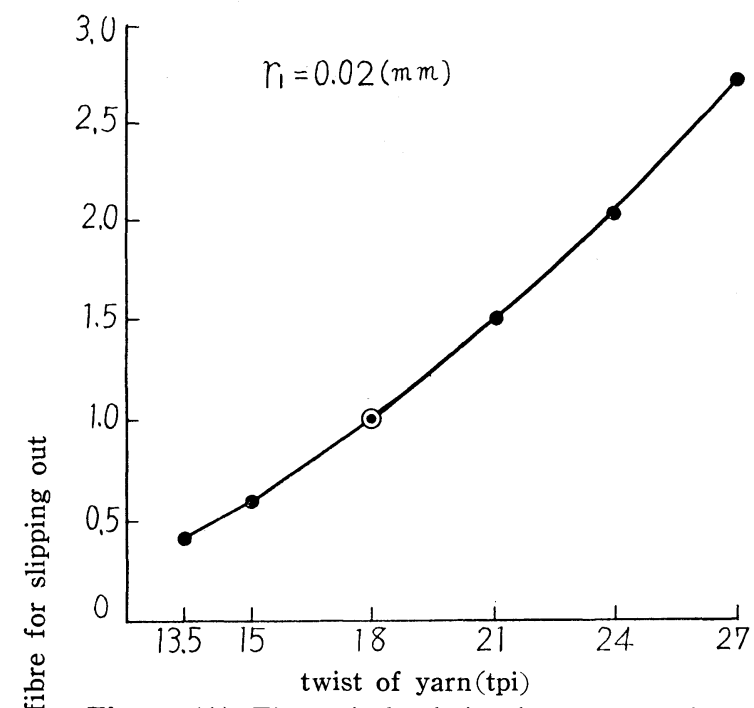

Fig. 16 (A) Theoretical relation between number of twists of yarns and relative resistance power of fibres against slippae out of yarns

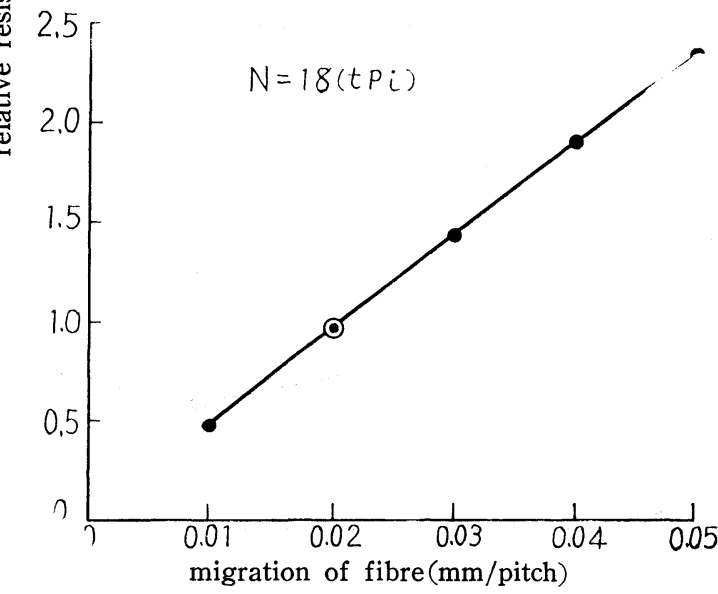

Fig. 16 (B) Theoretical relation between migration of fibres and relative resistance power of fibres against slippage out of yarns yarns $r_{1}=0.02 \mathrm{~mm}, N=18 \mathrm{tpi}$ is 1 . The relative resistance power of fibers $3 \mathrm{~mm}$ long against slippage out of various yarns - based on this assumption - is given in Figs. $16(\mathrm{~A})$ and (B).

The relation between the theoretical relative resistance power of fibers against slippage out of 30 's rayon staple fiber yarns $r_{1}=0.02 \mathrm{~mm}$ and the fuzz generated by the experimental rubbing is obtainable by combining Figs. 15 (A) and (B) and Figs. 16 (A) and (B).

Fig. 17 shows the theoretical relative anti-slippage resistance power of fibers and fuzz generated by rubbing $0,10,20,30$ and 40 times.

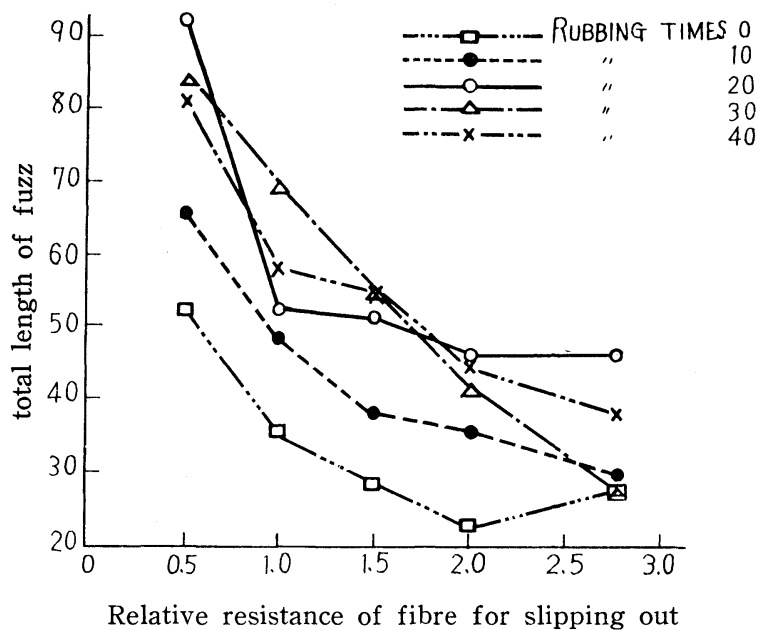

Fig. 17 Relation between relative resistance power of fibres against slippage out of yarns and total length of fuzz which is obtained from Figs. 24 (A) and $30(\mathrm{~A})$

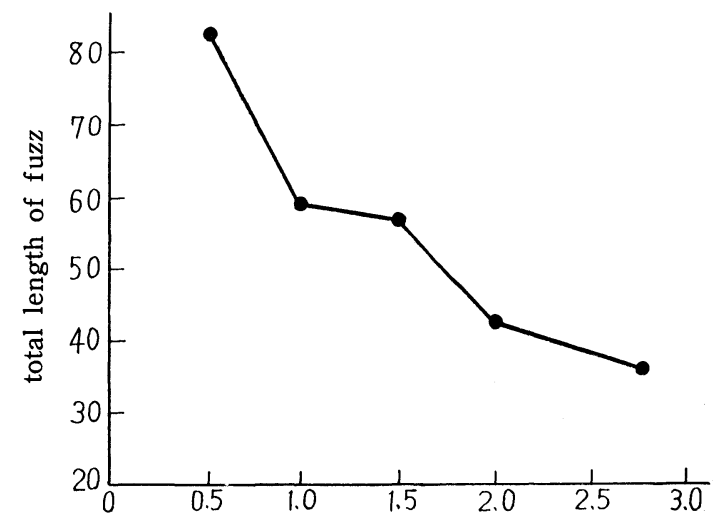

Relative resistance of fibre for slipping out

Fig. 18 Relation between relative resistance power of fibres against slippage out of yarns and total length of fuzz obtainable from Fig. 24(B) and Fig. $30(\mathrm{~A})$

Fig. 18 shows the relation between the theoretical anti-slippage reisistance power of fibers and the mean 
quantity of fuzz generated by rubbing 10-40 times.

The 2 figures show that the total length of fuzz generated by rubbing is not directly in inverse ratio to the theoretical anti-slippage resistance power of fibers. Instead, fuzz increases rapidly in total length as the theoretical resistance power of fibers decreases. The reason presumably is that, as fibers are pulled out and fuzz gets longer, the force of outside friction which pulls out fibers increases and helps the generation of fuzz by rubbing.

It is next to impossible to spin test yarns having the same number of twists and varying in the degree of migration. However, the relation between fiber migration and the degree of the tendency of fuzz to generate can be estimated by using - as shown in Fig. 19 - the relation between the theoretical antislippage power of fibers and the experimental genera. tion of fuzz.

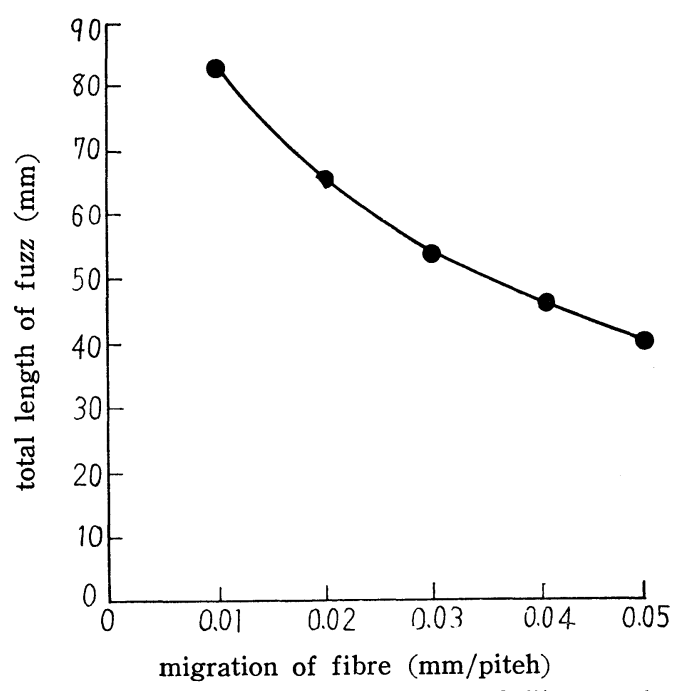

Fig. 19 Relation between migration of fibers and total length of fuzz

Similarly, it is possible to estimate the relation between the fuzz-generating tendency of yarns and other factors in the resistance of fibers to pulling out - the diameter of fibers, the length. frictional coefficient $^{-}$and diameter of yarns, etc.

However, the bearings which fiber fineness and length have on the fuzz-generating tendency vary with the tensile strength and abrasion resistance of fibers.

\section{Conclusions}

The tendency of fuzz to generate in yarns has an important bearing on weaving, knitting and the utility value of products made of yarns. The tendency can be estimated from the resistance power of fibres against slippage out of yarns. This article measures theoretically the resistance power of fibres against slippage out of yarns from the geometrical construction of yarns.

It also reports on experimental generation of fuzz in rayon spun yarns, with $2 \mathrm{~d} 60 \mathrm{~mm}$ fibres, varying in the number of twists. The results of the experiments prompt these conclusions :

(1) There is a high correlation between the relative resistance power of fibres against slippage out of yarns, (the resistance power is measurable from the geometrical structure of yarns) and the total length of fuzz generated in our experiments. The tendency of fuzz to generate in yarns can, therefore, be estimated from their geometrical structure.

(2) It is difficult for fuzz to generate if the coefficient of friction and the crimps of fibers increase. Fuzz generates easily if the yarn diameter increases. If fibers are long, hairs with on end free decrease in number, but all hairs grow longer.

(3) It is difficult for fuzz to generation if fiber migration and the number of twists of yarns increase. In a yarn made up of fibers of an equal length and an equal thickness, fuzz generates easily if the yarn increases in thickness.

\section{Literature cited}

[1] W. J. Onions, J. Pickering, W. Stables ; J. Text. Inst., 41, P 480 (1950)

[2] W. J. Onions, M. Yate; J. Text. Inst., 45, T873 (1954)

[3] Lappage and Onions; J. Text. Inst., 55, T 381 (1964)

[4] A Barella; J. Text. Inst., 45, P 699 (1954)

[5] A Barella; Bull. Inst. Text. France, P 45 (1958-8)

[6] Centre de Recherches des Industries Textile de Rouen; "1" Bull. Inst. Text. France, p. 33 (1955), “2”, p. $37(1962-1 / 2)$

[7] H. R. Boswell, P. P. Townend ; J. Text. Inst., 48, P 135 (1957)

[8] Mihira, Sugimoto; Society of Fibre Science and Technology, Japan, 19, P. 43 (1963)

[9] K. Tanaka; J. Text. Mach. Soc. Japan, 17, p 783 859 (1964), 18, p. 103 (1965) 